\title{
Desarrollo Empresarial y Erradicación de Cultivos ilícitos: caso vereda Nueva Colombia departamento del Meta Colombia
}

\section{Business development and eradication of illicit crops: the case of vereda Nueva Colombia department of Meta Colombia}

\author{
Guillermo Mendivil-Gonzalez \\ Escuela Naval de Cadetes Almirante Padilla- Colombia \\ ORCID iD: https://orcid.org/0000-0002-7601-0999 \\ guillermo.mendivil@armada.mil.co \\ Jaime E. Gonzalez-Díaz* \\ Escuela Naval de Cadetes Almirante Padilla- Colombia \\ ORCID iD: https://orcid.org/0000-0002-9441-5543 \\ poseidon@enap.edu.co
}

\author{
José D. Peroza-Daza \\ Escuela Naval de Cadetes Almirante Padilla- Colombia \\ ORCID iD: https://orcid.org/0000-0002-1758-2380 \\ dfcn@enap.edu.co
}

* Autor a quien debe ser dirigida la correspondencia
Fecha de recepción: 26/09/2021

Fecha de evaluación: $10 / 10 / 2021$

Fecha de aceptación: 03/11/2021

Cómo citar: Mendivil-González, G., González-Díaz, J., \& Peroza-Daza, J. (2021). Desarrollo Empresarial y Erradicación de Cultivos ilícitos: caso vereda Nueva Colombia departamento del Meta Colombia. Revista Cientifica Anfibios, 4(2), 72-83. https://doi.org/10.37979/afb.2021v4n2.97

\section{Resumen}

Atribución-NoComercial-CompartirIgual 4.0 Internacional

Este artículo tiene como objetivo identificar la relación entre el desarrollo empresarial y la estrategia de la erradicación de cultivos ilícitos, por medio de una propuesta para la Vereda Nueva Colombia Departamento del Meta Colombia. Este trabajo se basó en un estudio con un diseño documental. Este texto es producto de una investigación de diseño documental, de carácter descriptivo, y finalmente, es proyectiva pues presenta una propuesta de solución a la problemática identificada. El artículo inicialmente hace un recorrido histórico del problema del narcotráfico en Colombia durante la segunda mitad del siglo XX, describiendo los eslabones de la cadena productiva del negocio de las drogas ilícitas en el contexto global, para posteriormente presentar la evolución de las políticas públicas en el país enfocadas a la erradicación de cultivos ilícitos, consecuentemente presenta un análisis teórico del constructo desarrollo empresarial, para finalizar con una propuesta de aplicación de este a la erradicación de cultivos, la cual la enfoca en cuatro ejes: dialogo entre instituciones sociales y creación de tejido social, presencia del estado, mejoramiento de infraestructura vial y productiva, y consolidación de procesos productivos, su comercialización y valor agregado.

\section{Palabras clave}

Desarrollo empresarial; erradicación; cultivos ilícitos; narcotráfico; drogas

\begin{abstract}
This paper aims to identify the relationship between business development and the strategy for the eradication of illicit crops, through a proposal for the Vereda Nueva Colombia Department of Meta Colombia. This work was based on a study with a documentary design. This text is the product of a descriptive documentary design research, and finally, it is projective as it presents a proposed solution to the identified problem. The article initially makes a historical overview of the drug trafficking problem in Colombia during the second half of the 20th century, describing the links in the productive chain of the illicit drug business in the global context, to later present the evolution of public policies in the country focused on the eradication of illicit crops, consequently it presents a theoretical analysis of the business development construct, to end with a proposal for its application to the eradication of crops, which focuses on four main theme: dialogue between social institutions and creation
\end{abstract}


of tissue social, state presence, improvement of road and productive infrastructure, and consolidation of productive processes, their commercialization and added value

\section{Keywords}

Business development; eradication; illicit crops; drug trafficking; drugs

\section{Introducción}

El comercio internacional de drogas se basa en un negocio con una fuerte estructuración de productores y consumidores, de un lado algunos países de bajos ingresos dedicados a la producción y oferta de grandes cantidades de drogas, y de otro lado, países ricos con una gran base de consumidores con capacidad de compra; es decir, si se mira el problema de los dos lados, se está hablando de un problema global que afecta a países ricos y pobres, desde diferentes eslabones de la cadena de un millonario negocio.

Adicionalmente, hay que tener en cuenta que el problema global de drogas ha tenido un protagonismo en la escena mundial de los últimos sesenta años, y es tal la magnitud del mismo, que se debe analizar desde diferentes perspectivas políticas, jurídicas e institucionales. Todo lo anterior ha generado la creación de un sistema mundial de seguimiento, inspección, y penalización para hacer frente a los actores criminales que lideran el negocio transnacional de las drogas; más aún, cuando esta actividad ilegal profundiza flagelos sociales como la violencia, las adicciones, la delincuencia común y organizada, ligados directamente al comercio mundial de drogas (Ahumada, 2019).

Colombia ha sido un protagonista en el comercio mundial de las drogas ilícitas, principalmente en el procesamiento y la exportación de alucinógenos, esto provocado por un lado, y como ya se señaló, por la alta demanda de este producto en países de altos ingresos, y ayudado por la incapacidad del Estado para poder controlar zonas que por diversas razones se constituyen en puntos clave para el cultivo, procesamiento, y distribución de estas mercancías.

En varios países el negocio de las drogas ha posibilitado el agravamiento de problemas como la violencia; sobre todo porque las jugosas utilidades de esta actividad ha permitido la compra de grandes cantidades de armamentos para diferentes grupos al margen de la ley, pero este es solo uno de los problemas causados por la cadena de la producción de droga, ya que este también ha desembocado en problemas de tipo ambiental, económico y político. El desarrollo de toda esta economía ilegal en Colom- bia ha seguido diferentes periodos; el primero fue la bonanza marimbera, el cual tendió el puente para lo que sería el auge de la producción de cocaína, y el surgimiento de los grandes carteles de la droga, hasta nuestros días.

El primer gran periodo del auge del negocio de la droga en Colombia se conoce como la bonanza marimbera. Se gestó principalmente en la costa caribe a mediados de la década de 1970, y principios de la década de 1980, con grandes extensiones cultivadas de marihuana (Cannabis sativa), que tenían como destino mayoritariamente los consumidores de Estados Unidos. El impulso que tuvo la siembra de la marihuana, tuvo como impulsor entre otras causas la decadencia de la producción algodonera, lo cual permitió que el campesinado viera en este cultivo ilegal una alternativa para sus menguadas economías.

Así las cosas, inicia la lucha contra el cultivo, y la comercialización de la marihuana, por lo cual el narcotráfico migro al negocio de la cocaína, el cual vino de la mano con el surgimiento de los grandes carteles de la droga, con lo cual para la década de 1990, ya se había constituido la bonanza de la pasta de coca procedente de Perú y Bolivia. A diferencia de la bonanza de la marihuana en donde Colombia solo cultivaba, mientras su comercio y distribución estaba en manos de las mafias de Estados Unidos; en la bonanza de la pasta de coca los narcotraficantes colombianos dominaron la producción, y el tráfico transnacional del mismo.

Más adelante, entraron los grupos guerrilleros principalmente las FARC- a jugar un rol protagónico brindando vigilancia a los cultivos ilícitos, en lo que encontraron un jugoso medio de financiación, con el que incrementaron su poder armado, y capacidad bélica. Como respuesta a esto, surgieron grupos paramilitares para defender a los terratenientes que eran los principales objetivos de las guerrillas. En este contexto, y producto de la guerra contra los capos de la droga, los carteles fueron diezmados, al tiempo que Perú disminuyo ampliamente su cuota en el cultivo de coca; lo cual dio pie a que las guerrillas y los grupos paramilitares se apoderaran del negocio desde el cultivo, hasta la comercialización de la coca. 
Con este panorama inicio el país el siglo XXI, unos grupos al margen de la ley fortalecidos, y controlando el negocio de las drogas ilícitas, con un incremento en las áreas sembradas, y con el control de otros eslabones del comercio de drogas ilícitas, lo cual ha degenerado en incremento de sus actos violentos, y destrucción de lo social, y ambiental. Como estrategia para atacar las finanzas de estos grupos armados, se empieza a atacar el problema desde el cultivo mediante la erradicación de cultivos ilícitos mediante la fumigación con glifosato, y la erradicación manual forzosa (Gil y Villalobos, 2015).

Por otro lado, hay que destacar que el negocio de las drogas es una cadena de valor agregado que comienza con el cultivo, continua con la transformación primaria de hoja de coca a pasta básica y base de cocaína, y concluye con la comercialización y distribución. De toda la cadena, el transporte es la etapa más complicada, pues exige un entramado criminal conformado por diferentes actores con asignaciones muy específicas. Anteriormente un narcotraficante controlaba todo el proceso desde el cultivo, pasando el proceso de transformación, hasta finalizar con la distribución; hoy día esta cadena es responsabilidad de diferentes organizaciones muy especializadas (Cajiao, et al, 2018; Mejía, y Rico, 2010; Fonseca, et al. 2019).

Retomando el tópico de la lucha contra el narcotráfico, y teniendo en cuenta la cadena productiva del negocio de las drogas, uno de los eslabones en que se ha concentrado esta batalla es el cultivo, de tal suerte que un grueso de la estrategia se ha centrado en la erradicación de cultivos ilícitos; lo que ha mostrado la historia es que mientras se erradica una hectárea de coca se siembra otra, esto obedece a múltiples factores como crisis económica, marginalidad y violencia en el campo colombiano (Ortiz, 2003). Así pues, este artículo intenta presentar una reflexión alrededor del desarrollo empresarial como alternativa para que la erradicación de cultivos ilícitos logre una mayor efectividad.

\section{Metodología}

Este texto es producto de una investigación de diseño documental, ya que analiza una gran cantidad de artículos, boletines, y otros reportes de investigación, sobre la temática en cuestión
(Peña y Pírela, 2007; Maass, 2005). Igualmente, tiene carácter descriptivo, puesto que presenta una caracterización de los fenómenos desarrollo empresarial, y política de erradicación de cultivos, otorgándole un matiz diagnóstico. Finalmente, es proyectiva pues presenta una propuesta de solución a la problemática identificada (Hurtado, 2010). La recolección de la informacion se realizó por medio de fichas de investigación, y su procesamiento se realizó por medio del análisis del discurso.

\section{Resultados}

\section{Desarrollo empresarial y la estrategia de erradicación de cultivos ilícitos}

Como todo negocio, los cultivos ilícitos tienen su motivación en la expectativa de obtener una ganancia que justifica el riesgo, igualmente, como todos los negocios, nacen con la iniciativa de un emprendedor que encuentra en la posibilidad de generar riqueza el impulso para asumir el riesgo de emprender. Así lo señala la Comisión Europea, quien en su libro verde reconoce el espíritu empresarial como un catalizador de la innovación, la competitividad y el crecimiento (Pieschacón, 2006). Es decir, se hace necesario presentar a estos empresarios del campo, que apuntan su iniciativa en los cultivos ilícitos, una alternativa que les genere iguales o mejores ganancias, para hacer de la estrategia de erradicación una que alcance una alta efectividad. Pero, antes de cualquier propuesta se realizara un análisis a la evolución de la política nacional de erradicación de estos cultivos.

\section{Evolución de la política nacional de erradicación de cultivos ilícitos}

En el marco de la guerra mundial contra las drogas, y siendo Colombia protagonista de la misma; apurado por presiones internacionales, asume como una de sus estrategias la erradicación de cultivos ilícitos. Desde finales de la década de 1960 que se empieza a proponer esta estrategia, el país se ha debatido entre la erradicación forzosa y la erradicación manual. Inicialmente, se aplicó la erradicación forzosa. Posteriormente, se disminuyen las fumigaciones y se incrementa la erradicación manual, pero siempre regresando a la erradicación forzosa por considerarse más eficiente (Gil y Villalobos, 2015). 
Todo inicia bajo el gobierno de Misael Pastrana Borrero (1970-1974), quien establece un convenio con el Gobierno de los Estados Unidos Para la erradicación de las dogas, ya el presidente Richard Nixon se había comprometido con su país a lo que se conoce como la lucha contra las drogas. Es el mismo Pastrana quien expide el Estatuto Nacional de Estupefacientes, y quien también firma el Acuerdo Suramericano sobre Estupefacientes. Pero, fue durante el gobierno del presidente Julio Cesar Turbay (1978-1982) que inicia en el país esta estrategia con la fumigación de los cultivos de marihuana de la costa utilizando Paraquat un herbicida de alta toxicidad para el consumo humano.

Seguidamente, ya en la década de 1980, durante el período del presidente Belisario Betancur (1982-1986) se continuaría con la erradicación forzosa, pero esta vez con Glifosato para la erradicación de los cultivos de marihuana y los nacientes de cocaína. Posteriormente, durante el gobierno del presidente Virgilio Barco (1986-1990), se disminuyen las fumigaciones, y se incrementa la erradicación manual (Zambrano, et al 2017).

Posteriormente, ya en la década de 1990, se comienza con la presidencia de Cesar Gaviria (1990-1994), se incrementó la erradicación de cultivos en las zonas del país donde sembraban coca y amapola iniciando las fumigaciones con glifosato. Acto seguido, durante la presidencia de Ernesto Samper Pizano (1994-1998), el distanciamiento con la política antinarcóticos de los Estados Unidos del presidente Gaviria, dio paso a un quiebre completo con la llegada de Samper, todo esto producto de las imputaciones sobre la financiación de su campaña con dinero de los carteles de la droga, en medio de todo esto Colombia se convirtió en el productor número uno de coca a nivel mundial.

El fin de siglo XX, y comienzo del nuevo milenio, se dio con la presidencia de Andres Pastrana (1998-2002), durante este periodo el país Colombia se sometió a las exigencias de los Estados Unidos se compromete a llevar a cabo el Plan Colombia para la lucha contra el narcotráfico, el principal componente de este plan fue de carácter militar, pero tuvo un componente importante de erradicación mediante fumigaciones con glifosato. Este plan continúa bajo la presidencia de Álvaro Uribe (2002-2010), y fortalece erradicación por medio de la aspersión con glifosato. El go- bierno de Juan Manuel Santos (2010-2018), ha propuesto un viraje de enfoque para comprender el problema mundial de drogas, indicando que el consumo no es un abuso sino un problema de salud pública, lo cual resulto en un combate menos agresivo de los cultivos ilícitos (Zambrano, et al 2017).

\section{La naturaleza del desarrollo empresarial}

Los estudios sobre la función empresarial han tenido gran desarrollo en los últimos treinta años. Mucho de esto centrado en la persona del empresario como gestor principal de crecimiento y desarrollo en la economía global. Ahora, si se parte de la concepción que una economía progresa cuando su sector empresarial tiene los estímulos que proponen principalmente sus gobiernos nacionales y locales, es innegable el efecto de lo primero sobre lo segundo (Weneker y Thurik, 1999, Audretch y Thurik 2001, Santos 2004; Carlsson, 1996; Carree et alia, 2001; Cappellin, 1992; Guzmán, et al, 2004; Díaz, et al. 2019).

El tema del desarrollo siempre ha constituido una preocupación permanente en los estudios políticos, sociales, y económicos. Sin embargo, existe un debate sobre el desarrollo y el crecimiento económico, que en el plano de la empresa se hace más confuso. Lo que si queda claro es que existe una delgada línea que divide los dos constructos. Para el caso del desarrollo al nivel de la empresa, se puede decir que hace referencia al conjunto de acciones, que permiten el uso y la adquisición de capacidades y recursos empresariales, para encontrar nuevas opciones, nuevas soluciones, innovar; para lograr el cambio; que a su vez, resulta en mejores procesos y resultados que coadyuven al crecimiento sostenible de la empresa (Varela y Bedoya, 2006; Martínez, 2006; Aleán, 2006; Castro, 2007; Díaz, 2014).

De igual forma, el desarrollo empresarial se puede ver desde tres perspectivas: el ciclo de vida de la empresa, los recursos y capacidades, y la motivación. La primera, hace referencia a la evolución de la empresa por etapas: introducción, crecimiento, madurez, y declive. La segunda, está centrada en la existencia de recursos y capacidades sobrantes, que le brindan ventajas competitivas a la empresa. Y finalmente, la perspectiva de la motivación centrada más en la actitud individual de algunos empresarios, y de cómo asumen 
mayores riesgos en la toma de decisiones (Blázquez, et al, 2006; Díaz, 2014; Whetten, 1987; Boulding, 1950; Canals, 2000; Abad, 1996; Miner, 1980 y 1990; Davidsson y Wiklund, 1999).

De otro lado, el desarrollo empresarial tiene varias áreas de actuación como: la creación de empresas, el fortalecimiento empresarial, la formalización de empresas, y las redes empresariales. La primera, hace referencia al proceso emprendedor, que va desde la gestación de la idea, la búsqueda de financiación, hasta el montaje de la firma. La segunda, con un énfasis en el apoyo principalmente a las pequeñas y medianas empresas dada la importancia de estas en el tejido empresarial de muchos países.

La tercera área de actuación, son las política para las empresas informales, que a pesar de no estar reconocidas, y no estar reguladas, tienen un papel protagónico sobre todo en las economías del tercer mundo, donde aportan casi el $70 \%$ de los puestos de trabajo; así las cosas, es vital fortalecer estas unidades productivas, aunque si ben la formalización de empresas no es todo, es un inicio necesario en ese sentido. Finalmente, reconoce la importancia de desarrollar un ecosistema empresarial donde tienen gran valor los clúster, las cadenas productivas, o los distritos industriales (Díaz, 2014; Gatto, y Jos, 1997; Blázquez, et al, 2006).

Ahora, es importante delimitar los niveles en que interviene el desarrollo empresarial, los cuales son: el nivel de la economía o nivel macro, y el nivel empresarial o nivel micro. El primero, es el nivel económico o macro, hay que entender que aun que son las empresas las que compiten, son las regiones las que proporcionan los entornos favorables en los que las empresas forman y fortalecen sus capacidades. Para que un país o región pueda facilitar el desarrollo de su sector empresarial se deben establecer condiciones positivas desde el marco macroeconómico, regulatorio y de políticas e incentivos, el sistema financiero y mercado de capitales, el sistema de innovación y desarrollo tecnológico, el recursos humanos, el sistema institucional de apoyo técnico, gestión empresarial y cooperación entre firmas y la infraestructura física y territorio (Gatto y Jos, 1997; Vergara, 1999; Díaz, 2014).

En segundo término, está nivel micro o nivel empresarial, se hace desde dentro de la empresa, que puede financiar con recursos propios o por aportaciones adicionales de los socios para una mejora de la producción, adquisición de tecnología, capacidad de distribución y ventas, etc. Algunas formas de desarrollo empresarial del nivel micro son las mismas estrategias empresariales: desarrollo de mercado, desarrollo de producto, penetración, diversificación, etc.

El desarrollo empresarial señala las acciones, que posibilitan adquirir y utilizar recursos y capacidades para la innovación y la mejora, en procura de un crecimiento sostenible de la organización. El desarrollo empresarial se puede comprender desde tres perspectivas: el ciclo de vida de la empresa, los recursos y capacidades, y la motivación. Igualmente, interviene en el mundo económico desde los niveles macro y micro; en diferentes áreas de trabajo como: la creación de empresas, el fortalecimiento empresarial, la formalización de empresas, y las redes empresariales.

Teniendo claro que el desarrollo empresarial le apunta a la innovación, y la mejora, para el crecimiento sostenible de los negocios; y también, sabiendo que es el empresario la génesis y motor del desarrollo empresarial. Así pues, habría que señalar que este constructo aplicado al negocio de los cultivos ilícitos, en procura de hacer más efectiva la política de erradicación de estos cultivos. Tendría que presentar una alternativas tanto a nivel macro como micro, que permitan de forma innovadora utilizar sus recursos y capacidades para orientarse a nuevas opciones de mercado. En este artículo, se intenta proponer una alternativa desde el desarrollo empresarial para la sustitución de cultivos ilícitos en el caso de la Vereda Nueva Colombia, Departamento del Meta.

\section{Situación actual de los cultivos ilícitos en la Vereda Nueva Colombia}

La Vereda Nueva Colombia es una de las 84 veredas que constituye el municipio de Vista Hermosa en el Departamento del Meta. El municipio está ubicado a dos horas y media de Villavicencio capital del departamento, tiene una población de 25,000 pobladores, colinda al norte con el Municipio de San Juan de Arama Nororiente: Municipio de Puerto Lleras, al Sur; con el Departamento Del Guaviare y Municipio de La Macarena, al sur occidente; con el Municipio de Uribe, por el 
oriente; con el Municipio de Puerto Rico, y por el occidente; con el Municipio de Mesetas.

La región cuenta dentro de su subsistema biofísico con un importante recurso hídrico conformado por tres cuencas hidrográficas así: Río Ariari, Río Duda, y Río Guayabero. Igualmente, esta zona posee una amplia variedad de atractivos naturales entre los que se puede mencionar: los termales en la vereda termales, las playas del rio Guejar, la mina de asfalto en Maracaibo, los chorros de Sardinata en la vereda Maracaibo, los chorros Unión en la vereda de Caño Unión, el parque arqueológico en la vereda Cunimia y los atractivos del Parque Natural Nacional Serranía de La Macarena. El turismo tendría gran apoyo en la construcción de miradores turísticos en Piñalito y Vista Hermosa, y un parador turístico en Puerto Lucas (Alcaldía de Vistahermosa, 2018.)

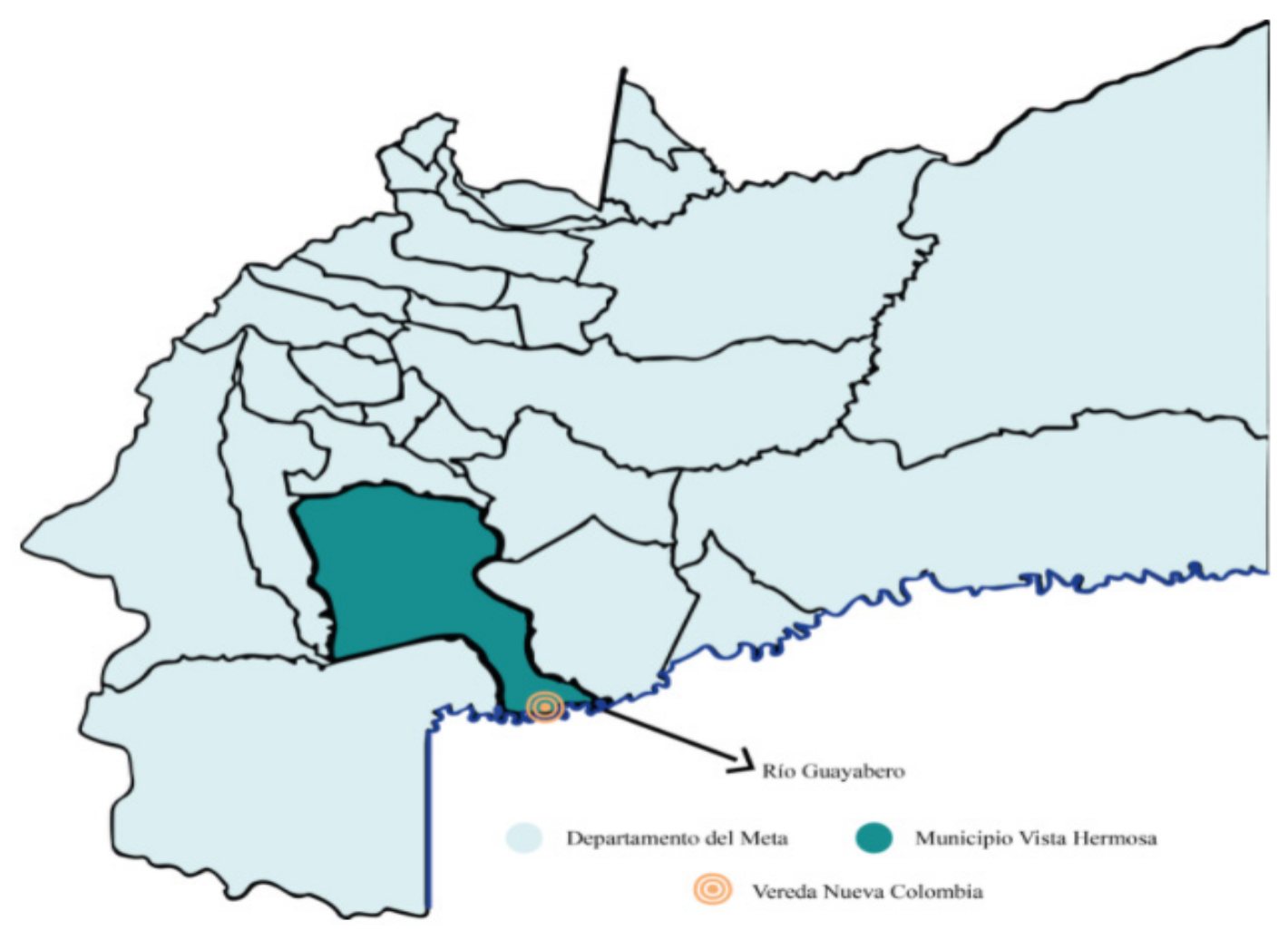

Imagen 1. Vereda Nueva Colombia, Departamento del Meta

Fuente: Verdad Abierta (2020)

Ya en la década de 1980, la coca había echado raíces, extinguió las plantaciones campesinas, y desarrollo una economía que incluso imponía un sistema de impuestos por los grupos al margen de la ley -principalmente las FARC-EP- gestando una gran influencia social y política. La situación ha cambiado con el tiempo, principalmente después de los acuerdos de paz; sin embargo, en la actualidad, la Vereda no están dentro del Programa Nacional Integral de Sustitución de Cultivos Ilícitos (PNIS), pese a que sus pobladores demuestran estar dispuestos a participar en la sustitución de cultivos, y la implementación de proyectos productivos alternativos factibles, pese a las amenazas de los Grupos Armados Organizados que aun delinquen en esa zona, y que les prohíben que se acojan a los progra- mas productivos del gobierno (Jiménez, et al, 2018; UNODC, 2019; Verdad Abierta, 2020).

\section{Propuesta de solución para la sustitución de cultivos ilícitos en la Vereda Nueva Colombia}

Frente a la problemática que vive la Vereda Nueva Colombia se propone una solución integral alternativa que involucre a todos los actores claves en la problemática: cultivadores, Estado, Fuerzas Armadas, instituciones sociales, Cámaras de Comercios (Ver figura 1). Lo anterior se deriva de la idea, que el problema del narcotráfico es un problema multifactorial que no debe ser visto desde una sola variable (el cultivo), sino que debe ser tratado de forma integral. Con esto como consigna 
se propone: dialogo entre instituciones sociales y creación de tejido social, la actuación del estado, consolidación de procesos productivos, mejora- miento de infraestructura vial y productiva, y comercialización y valor agregado.

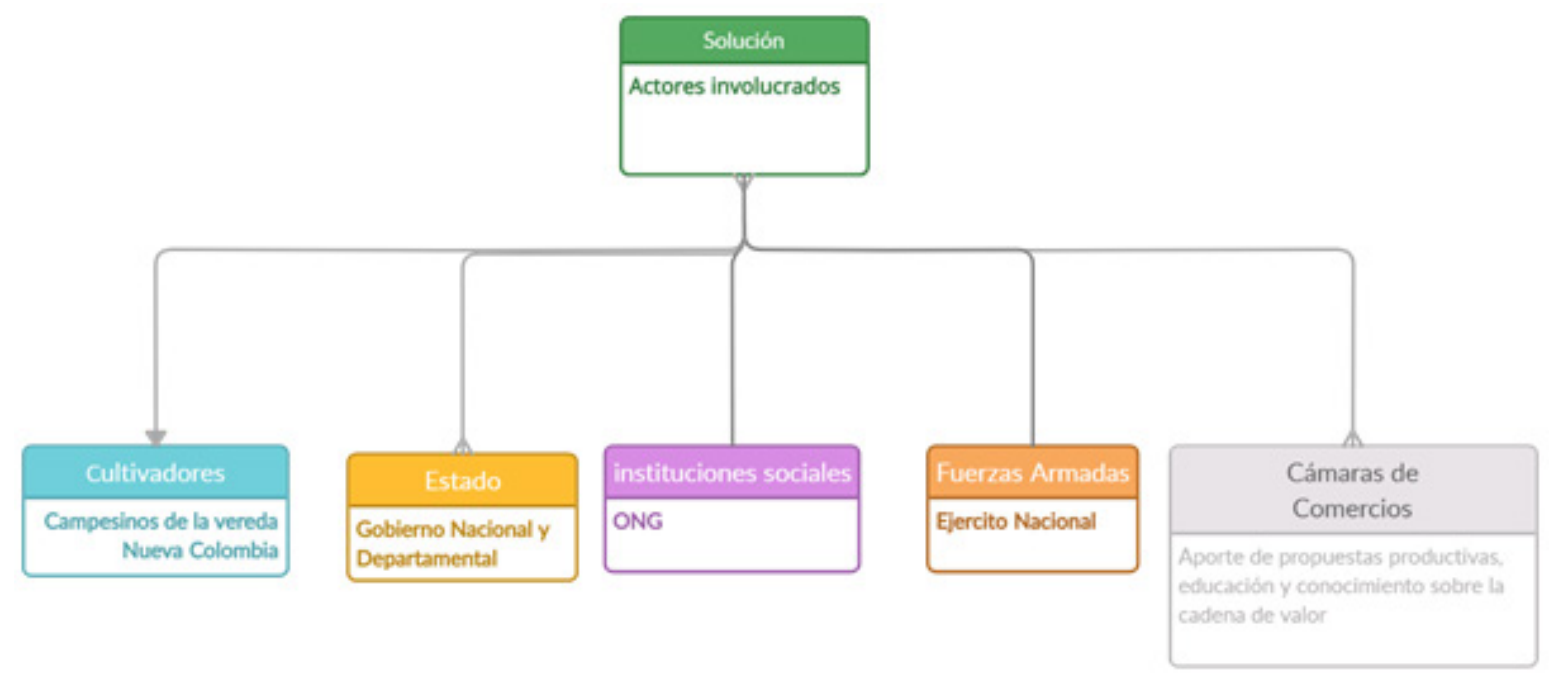

Figura 1. Actores involucrados en la gestión de soluciones para la vereda Nueva Colombia Fuente: elaborado por los autores

\section{Dialogo entre instituciones sociales y creación de tejido social}

Cualquier programa o proyecto que piense atender esta problemática, debe partir de los compromisos que deben asumir los diferentes actores que se ven implicados en un programa y hasta qué punto estos compromisos serán asumidos y ejecutados. Es decir; evidenciar la relación del programa de sustitución con el gobierno departamental y municipal, al igual que otras organizaciones de carácter local, regional e internacional, demuestra que cualquier estrategia exige y necesita de su apoyo para ser implementada adecuadamente, requiere percibir sus dinámicas internas y las dificultades frente a la comunidad para poder formular unos objetivos, claros, alcanzables, y verificables.

Juntas de acción comunal. Las Juntas de Acción Comunal son organizaciones cívicas comunitarias sin ánimo de lucro, integradas voluntariamente por residentes de un barrio que buscan unirse con fundamento en la democracia participativa. Las JAC se convierten en instituciones sociales que buscan impactar el proceso administrativo de desarrollo de las localidades. Son de alguna manera una voz legitimada, una "herramienta canalizadora" de la comunidad para presentar propuestas de un sector frente a los gobiernos locales y departamentales. Son una alter- nativa para lograr que se gestione las necesidades de los habitantes de un sector.

Consejos municipales de paz. La oficina tiene el compromiso de realizar los consejos municipales de paz, que es el espacio central donde convergen todas las organizaciones, instancias y mecanismos de participación en asuntos de paz en el territorio. Los consejos de paz propician un ambiente favorable para la articulación de estos mecanismos, al crear visiones estratégicas, encontrar puntos de conexión y falencias entre las acciones implementadas.

Mesa de articulación. Por otro lado, la Oficina de Paz y Posconflicto crea la mesa de articulación, espacio donde participan diferentes organizaciones, a fin de evaluar cómo se ha avanzado y que dificultades han tenido los programas y organizaciones que han venido trabajando en Briceño, buscando articular acciones para lograr un mayor impacto. La mayoría de estos programas cuentan con recursos del gobierno nacional o de cooperación internacional.

Fundación ideas para la paz. La Fundación Ideas para la Paz (FIP) es un centro de pensamiento independiente creado en 1999 por un grupo de empresarios colombianos. Su misión es propiciar la generación de conocimiento, proponiendo iniciativas, desarrollando prácticas y acompañando 
el proceso para la construcción de paz en todo el territorio colombiano.

Oficina de las naciones unidas contra la droga y el delito. La oficina de las Naciones Unidas contra la Droga y el delito ha venido apoyando al gobierno colombiano en la gestión de políticas públicas encaminadas a la reducción y eliminación de cultivos ilícitos. El desarrollo alternativo es un proceso dedicado a reducir o eliminar los cultivos ilícitos mediante la adopción de medidas de desarrollo rural expresamente concebidas con tal fin. Estas medidas buscan alcanzar un desarrollo sostenible en los territorios dónde se están implementando estrategias contra las drogas ilícitas, teniendo en cuenta las características socioculturales y dinámicas de los territorios y sus comunidades.

Parques nacionales naturales de Colombia. Dentro de sus funciones se encuentran: gestionar el Sistema de Parques Nacionales Naturales, así como reglamentar el uso y el funcionamiento de las áreas que lo conforman, según lo dispuesto en el Decreto -Ley 2811 de 1974, Ley 99 de 1993 y sus decretos reglamentarios.

EI ministerio de ambiente y desarrollo sostenible. Es el órgano encargado de gestionar todo lo referente al medio ambiente y de los recursos naturales renovables, para lo cual debe orientar y regular el ordenamiento ambiental del territorio y de definir las políticas y regulaciones en relación con la recuperación, conservación, protección, ordenamiento, manejo, uso y aprovechamiento sostenible de los recursos naturales renovables y del ambiente de la nación, con el fin de garantizar el desarrollo sostenible.

\section{La actuación del estado}

Para comprender la problemática que enfrenta el campesinado, es imperativo realizar un análisis del rol que desempeña el Estado en esta problemática y como podría impulsar estrategias de desarrollo para lograr disminuir los cultivos y aumentar el crecimiento social. El problema de los cultivos ilícitos se presenta fundamentalmente por una falta de presencia del Estado en el sector rural del país. La falta de inversión y presencia estatal en diferentes regiones del país ha generado el desmejoramiento de la calidad de vida de los campesinos. Dicha situación pone en problemas al Estado ya que, según la Constitución Política colombiana, el Estado debe velar por la vivienda digna, salud, sanidad y la igualdad de trabajo, dicho compromiso no es cumplido en muchos de los municipios con presencia de cultivos de coca. A raíz de lo mencionado, el Estado se enfrenta al problema de cumplir con las necesidades de los colombianos en muchos territorios (García y Vargas, 2018)

\section{Consolidación de procesos productivos}

En el componente productivo, se requiere sembrar productos agrícolas alternativos entre los que se podría sugerir: café, cítricos, cacao y frutales, también se deben fortalecer y conformaron las asociaciones de productores, comercializadores, de asistencia técnica y de distritos de riego. La asistencia técnica se realizó bajo la metodología de Escuelas de Campo, ECAS, incluyendo capacitación y asesoría para la producción con abonos orgánicos para 242 grupos de trabajo. El programa debe incluir un componente financiero, que permita el acceso a créditos para las familias, y adicionalmente se deben proveer: insumos, herramientas, fertilizantes etc.

El municipio oferta variedad de paisajes y los atractivos del Parque Natural Nacional Serranía de La Macarena. Las grandes riquezas en materia de recursos naturales ofrecen a los visitantes la oportunidad de desarrollar actividades de ecoturismo, y de este modo generar de ingresos. El turismo se vería fortalecido con la construcción de miradores turísticos, recorridos turísticos, corredores turísticos, granjas de descanso, senderismo, entre otros. El ecoturismo es sostenible y rentable dado que muestra un crecimiento constante en la economía mundial debido a su gran margen de ganancia. Apostarle al ecoturismo no solo es una alternativa fuerte de desarrollo económico para la vereda.

\section{Mejoramiento de infraestructura vial $y$ productiva}

En el componente de infraestructura productiva se deben mejorar y construir vías rurales, las vías terciarias es un tema de enorme importancia que tiene todo tipo de beneficios para el desarrollo rural y la competitividad, ya que las deplorables condiciones de las carreteras provocan retrasos en el desarrollo agrícola. Creo que estas 
rutas para el campo son tan importantes o más importantes que la expansión de la línea principal o las rutas $4 \mathrm{G}$. La construcción de caminos terciarios confirma la necesidad del desarrollo de infraestructura vial en áreas rurales y su impacto en el crecimiento económico.

El desafío es desarrollar soluciones que garanticen la disponibilidad de infraestructura local. En este sentido, la construcción, mejora y mantenimiento de vías terciarias corresponden al objetivo de implementar medidas que promuevan el desarrollo local. Para que estas soluciones tengan resultados definitivos y un impacto social real, es necesario que las inversiones realizadas tengan en cuenta los elementos de adaptabilidad al cambio climático, riesgos naturales y seguridad vial, así como diversos proyectos sociales y productivos.

\section{Comercialización y valor agregado}

Es necesario generar alianzas comerciales entre los productores/beneficiarios y compradores o posibles compradores. Se sugiere la participación en rueda de negocios, ferias comerciales y reu- niones de compras interinstitucionales, entre estas: el Programa de Alimentación Escolar, PAE, promovida por la Gobernación del Departamento, el Instituto Colombiano de Bienestar Familiar y la Cámara de Comercio. Para la comercialización de café se puede desarrollar un acuerdo con la Federación de Cafeteros. También, se pueden ubicar puntos de compra en los diferentes municipios.

Para ello, deben reorientar las estrategias de mercadeo para con el fin de impulsar de forma casi inmediata los productos y sus recursos naturales, llevando así, ahora, la comercialización a las transacciones internacionales. Por eso, ha dado a ver los mejores y más rentables medios para la distribución de sus productos, creando grandes diferencias entre el producto procesado y el producto sin transformar; además modificar el consumo induciéndole al consumidor una necesidad que tal vez no tenia o quizás tenia y deciden que incrementar su importancia. Grandes cambios se han presentado y por eso, el sector agropecuario de Colombia ha tomado fuerza entre los empresarios e inversionistas.

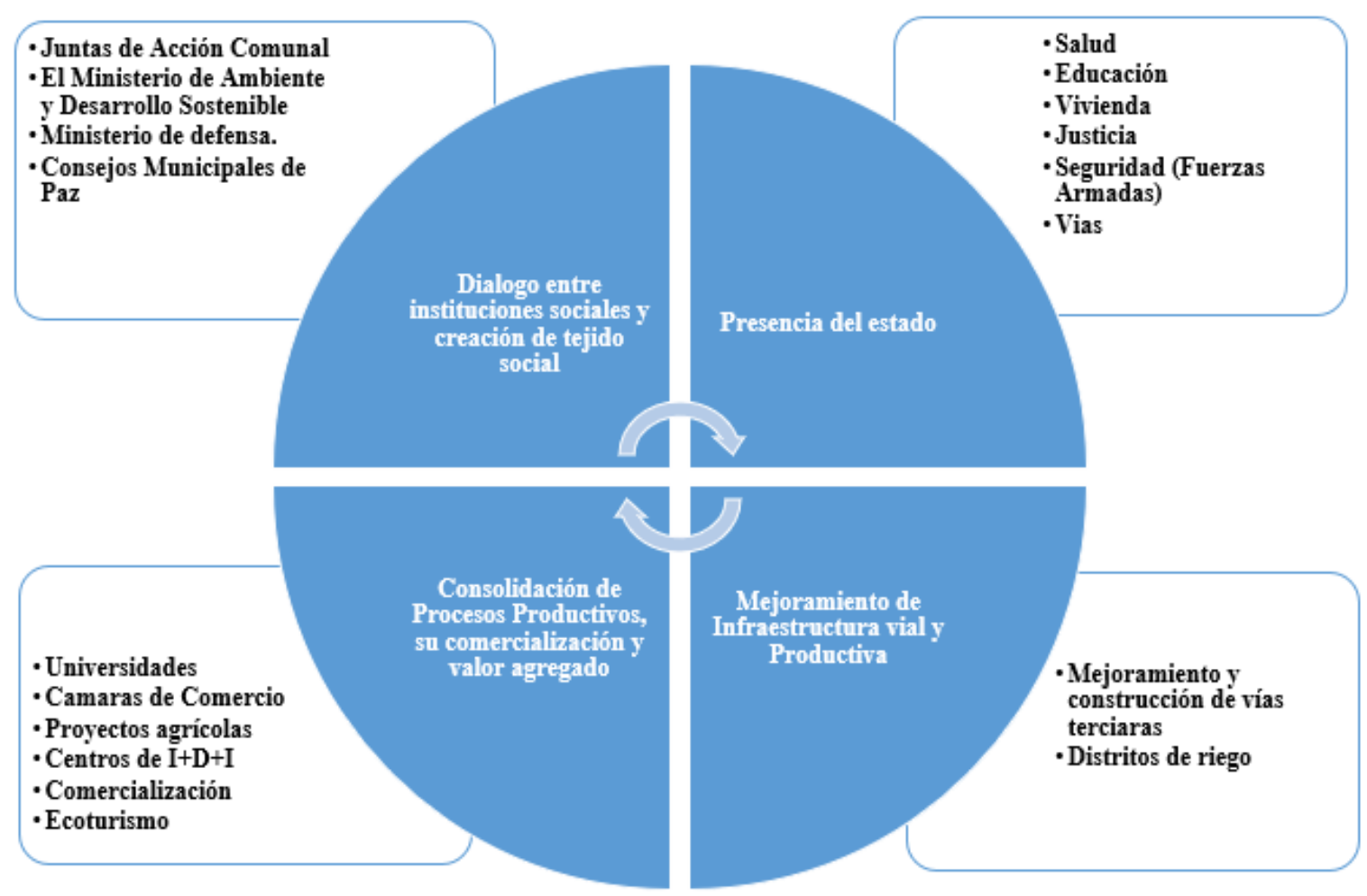

Figura 2. Propuesta de solución para la sustitución de cultivos ilícitos en la Vereda Nueva Colombia Fuente: elaborado por los autores 


\section{Conclusiones}

Con casi seis décadas de participación como protagonista en el comercio internacional de drogas, Colombia se ha visto inmersa en una lucha global contra este flagelo, que ha dejado en el país un rastro de violencia, adicciones, y delincuencia común y organizada, entre otros.

Teniendo en cuenta, que como muchos negocios legales, el negocio de las drogas es una cadena de valor internacional compuesta por el cultivo, la transformación, y la comercialización y distribución. Partiendo de esta realidad, y en muchos casos por presiones internacionales la participación del país en la lucha antes señalada se han enfocado en la erradicación de cultivos ilícitos.

Ahora, visto como negocio el narcotráfico en todos sus eslabones parte con la iniciativa de un empresario con la expectativa de ganancias, que en el narcotráfico son jugosas y de corto plazo. Para el caso de Colombia los empresarios del campo que desarrollan cultivos ilícitos tienen móviles adicionales como lo son un sector agrícola con crisis económica, marginalidad y violencia. Lo cual de los cultivos ilegales una opción muy atractiva que juega en contra de las expectativas de la política nacional de erradicación de estos cultivos.

La política nacional de erradicación de cultivos desde sus inicios se ha movido entre la erradicación manual, y la erradicación forzosa mediante aspersión de herbicidas, siendo esta última la de mayor aplicación por su eficiencia. Pero el atractivo de sus utilidades hace de estos cultivos un problema de nunca acabar. Por tal razón este artículo plantea que sumando a la erradicación se deben aplicar acciones de desarrollo empresarial que la complementen con innovación, y otras soluciones tanto a nivel macro como micro, que permitan hacer desestimular los cultivos ilegales.

Lo anterior se intenta demostrar con una propuesta alternativa desde el desarrollo empresarial para la sustitución de cultivos ilícitos en el caso de la Vereda Nueva Colombia, Departamento del Meta, partiendo del potencial agrícola y turístico de esta región se propone realizar un dialogo entre instituciones sociales que permita fortalecer el tejido social, fortalecer la presencia del estado, mejorar la infraestructura vial y productiva, y finalmente, consolidar los procesos productivos, su comercialización, y la generación de valor agregado.

\section{Referencias}

Abad. G. (1996). Los incentivos financieros y la motivación del personal: análisis empírico de las entidades financieras. Tesis de doctorado no publicada, Universidad de Málaga, Málaga, España.

Ahumada, G. (2019). América Latina y el Caribe en el Problema mundial de las drogas. In XIII Jornadas de Sociología. Facultad de Ciencias Sociales, Universidad de Buenos Aires.

Audretsch, D. B. y Thurik, A.R. (2000) "Capitalism and democracy in the 21st Century: from the managed to the entrepreneurial economy", Journal of Evolutionary Economics, 10, 17-34.

Blázquez. F. Dorta. J. Verona. M. (2006). Concepto, perspectivas y medida del crecimiento empresarial. Cuadernos de Administración, Pontificia Universidad Javeriana. Enero-junio, año/vol. 19, número 031. pp. 165-195.

Boulding, K. E. (1950). A reconstruction of economics. New York: Wiley.

Cajiao, A., González, P., Pardo, D., \& Zapata, O. (2018). Una aproximación al crimen transnacional organizado: redes de narcotráfico Colombia-España. Documento de trabajo, 5(15), 9.

Canals, J. (2000). La gestión del crecimiento de la empresa. Madrid: Mc Graw Hill.

Cappellin, R (1992): “Los nuevos centros de gravedad del desarrollo regional en la Europa de los noventa", Revista de Estudios Regionales, 33, 15-62.

Carlsson, B. (1996) "Small business, flexible technology and industrial dynamics" en P.H. Admiraal (Ed) Small business in the modern economy, De Vries lecturers in economics, Oxford: Balckwell Publishers, pp. 63-125. 
Carree, M., Van Stel, A. Thurik, R. y Wennekers, S. (2001) "Economic development and business ownership: an analysing using data of 23 OECD countries in the period 1976-1996", Small Business Economics, 19, 271-290

Davidsson, P. and J. Wiklund (1999). Theoretical and Methodological issues in the study of firm growth, Retrieved 10th November 2004 from:http://www.ihh.hj.se/eng/research/publications/ wp/1999-6\%20Davidsson,\%20Wiklund.pdf

Díaz, J. E. G., Cuisman, K., \& Hernández, P. D. (2017). Perdurabilidad empresarial de las mipymes de la ciudad de Cartagena: Un estudio de caso. In Congreso Internacional en Administración de Negocios Internacionales.: CIANI 2017 (pp. 335-354). Universidad Pontificia Bolivariana.

Díaz, J. E. G., Plaza, V. A. D., \& León, M. C. M. (2019). Critica a la mano invisible: Una propuesta de desarrollo alternativo para Colombia (No. 017112). Revista científica anfibios.

Díaz, J. G. (2014). Aproximaciones conceptuales al desarrollo empresarial. Aglala, 5(1), 86-106.

Enright, Michael (2001), "Regional Clusters: What we know and what we should know", taller Innovation Clusters and Interregional Competition, Kiel International, Institute 12 y 13 de November.

Fonseca-Moreno, A. C., Gualteros-Pinzón, C. E., \& González-Díaz, J. E. (2019). La minería criminal en el Río Atabapo frontera colombo venezolana: propuestas de solución. Revista científica anfibios, 2(2), 23-33.

García, M. Vargas, J. (2018) Sustitución o relocalización: opciones de política en zonas de cultivos ilícitos en Colombia. Trabajo de Grado. Facultad de Economía Universidad de los Andes.

Gatto, F. Jos, J. (1997). Guía metodológica para la preparación de estrategias de desarrollo empresarial y de la pequeña y mediana empresa. Banco Interamericano de Desarrollo. Washington, D.C. Tomado de: https://www.economia.gob.mx/pics/p/p2760/cipi_1Cguiametodologia-desemp.pdf. Consulta 18-02-09.

Gil, N. Villalobos, F. (2015). Análisis de la estrategia de erradicación manual forzosa en el municipio de Puerto Asís-Putumayo (2010-2014). Trabajo de grado para graduarse del Programa de Relaciones Internacionales y Estudios Políticos. Universidad Militar Nueva Granada. Bogotá

González-Díaz, J. E., Sánchez-González, J. A., Ochoa-De-Arco, E., \& Sánchez-Valbuena, I. (2019). Modelo de explotación pesquera sostenible como apuesta productiva: caso Moñitos-Córdoba-Colombia. Saber, Ciencia y Libertad, 14(1), 179-189.

Hurtado, J. (2010). Metodología de la investigación: guía para la comprensión holística de la ciencia. Quirón Ediciones.

Jiménez, S. Mendoza, M. Rodríguez, L. (2018). Café como alternativa de sustitución de cultivos ilícitos en el municipio de Mesetas Meta. Café como alternativa de sustitución de cultivos ilícitos en el municipio de Mesetas Meta. Trabajo de Grado para optar al título como especialista en Alta Gerencia y Economía Solidaria. Universidad Cooperativa de Colombia.

Maass, M. (2005). Laboratorio de investigación y desarrollo en comunicación compleja: una propuesta para pensar la complejidad. Andamios, 1(2), 79-96.

Martínez, B. (2006). Los factores que asfixian o instigan las innovaciones en las organizaciones. Tomado de: http://www.wikilearning.com/monografia/innovacion_en_las_organizaciones-barreras_culturales_al_desarrollo_empresarial/9665-3, Recuperado 21/01/2009

Mejía, D., \& Rico Valencia, D. M. (2010). The Microeconomics of Cocaine Production and Trafficking in Colombia (La Microeconomía De La Producción Y Tráfico De Cocaína En Colombia). Documento CEDE, (2010-19). 
Miner, J. B. (1980). A rationale for the limited domain approach to the study of motivation. En C. C. Pinder y L. F. Moore (Eds.), Middle range theory and the study of organizations (pp. 334336). Boston: Martinus Nijhoff.

Miner, J. B. (1990). Entrepreneurs, high growth entrepreneurs, and managers: Contrasting and overlapping motivational patterns. Journal of Business Venture, 5 (4), 221-234.

Miner, J. B., Crane, D. P. y Vandenberg, R. J. (1994). Congruence and fit in professional role motivation theory. Organization Science, 5 (1), 86-97.

Ortiz, C. E. (2003). Cultivos ilícitos y nueva ruralidad en Colombia. Cuadernos de desarrollo rural, (50).

Pieschacón. C. (2006). Empresa y bienestar social. Círculo de Empresarios de España. Madrid.

Santos, F.J. (2004): “Convergencia, desarrollo y empresarialidad en el proceso de globalización económica". Revista de Economía Mundial, Vol. 10-11.

UNODC (28 de julio de 2020). Informe de Monitoreo de Territorios Afectados por Cultivos Ilícitos en Colombia (2019). https://www.unodc.org/colombia/es/presentacion-informe-de-monitoreo-de-territorios-afectados-por-cultivos-ilicitos-en-colombia-2019.html

Verdad Abierta (13 de agosto de 2020). Campesinos de Vista Hermosa, bajo la estigmatización de la Fuerza Pública. https://verdadabierta.com/campesinos-de-vista-hermosa-bajo-la-estigmatizacion-de-la-fuerza-publica/

Vergara, R. (1999). "Estrategia para mejorar la Competitividad Regional. Seminario Estrategias para la Competitividad Regional en el Perú. Lima. Peru.

Wennekers, S., y Thurik, R. (1999): “Linking Entrepreneurship and Economic Growth”. Small Business Economics, 13.

Whetten, D. A. (1987). Organizational growth and decline processes. Annual Review of Sociology, $13,335-358$.

Zambrano, J., Ospina, G., Perilla, J., Zambrano, D. Ortiz, S. (2017). Colombia y la política de drogas: el policy-taking al policy-making a través de una aproximación desde la geopolítica crítica y el constructivismo en la política exterior. Universidad del Rosario. 